\title{
Anterior Hyaloidal Fibrovascular Proliferation (AHFVP) in a Diabetic after Cataract Extraction, Resulting in Hyphaema and Vitreous Haemorrhage during YAG Laser Capsulotomy
}

\author{
AO Hassan, ON Okonkwo, O Oderinlo, F Oluyadi , A Ogunro, A Harriman, R Duke \\ Eye Foundation Retinal Institute, Ijebu Imushin, Ogun State, Nigeria
}

\section{SUMMARY}

Aim: To report a rare case of anterior hyaloidal fibrovascular proliferation (AHFVP) which occurred after cataract surgery on a non-vitrectomized diabetic eye. This complication resulted in vitreous haemorrhage and hyphaema following $a$ $Y A G$ laser capsulotomy procedure.

Method: A case report of a patient managed at the above eye department. Clinical records as indicated in the patient's case note were reported.

Conclusion: Anterior hyaloidal fibrovascular proliferation is a complication which an eye surgeon should bear in mind when attending to diabetic eyes after cataract surgery. The potential danger of vitreous haemorrhage following YAG laser capsulotomy should also be borne in mind, as more centres in the country aspire to acquire a Y A G laser machine to perform capsulotomy.

Keywords: cataract surgery, anterior hyaloidal fibrovascular proliferation, $\mathrm{YAG}^{*}$ laser capsulotomy, diabetes mellitus

\section{INTRODUCTION}

Anterior hyaloidal fibrovascular proliferation (AHFVP) is a well recognized complication occurring in eyes that have undergone diabetic vitrectomy. ${ }^{1,2}$

It most commonly occurs after phakic vitrectomy and scleral buckling for diabetic traction retinal detachment. It usually manifests with haemorrhage into the vitreous cavity or anterior hyaloid 3 to 12 weeks after vitrectomy and is the result of fibrovascular proliferation from the peripheral retina extending toward the equator of the lens and on to the

\footnotetext{
" YAG = yttrium aluminium garnet, a crystal used in some types of laser for laser surgery.
}

posterior lens capsule., ${ }^{3,4}$ Patients who develop AHFVP typically have long-standing diabetes, extensive retina ischaemia and florid neovascularization despite panretinal photocoagulation.

Although AHFVP is commonly seen in post vitrectomized diabetic eyes, it is a rather uncommon occurrence after a cataract extraction. Medline search revealed only one reported case series of AHFVP which occurred after cataract surgery with implantation of a posterior chamber intraocular lens in four patients. ${ }^{4}$

Anterior hyaloidal fibrovascular proliferation occurs in the retrolenticular or ciliary body region., ${ }^{5,6}$ Typically, fibrovascular proliferation in ischaemic diabetic eyes is known to occur predominantly in the region posterior to the equator, ie, the pre-equatorial fundus. ${ }^{5}$ There is a preponderance of posterior neovascularization occurring in proliferative diabetic retinopathy. ${ }^{7,89}$ This posterior proliferative disease in ischaemic diabetic eyes, in turn, could result in vitreoretinal tractional forces, giving rise to the well known tractional or tractional-rhegmatogenous retinal detachment of the posterior pole seen in proliferative diabetic retinopathy. Fibrovascular proliferation in proliferative diabetic retinopathy occurring in the pre equatorial fundus, ie, the fundus anterior to the equator, is a more unusual finding. ${ }^{5}$

The case being reported is unique for two reasons. Firstly, the patient suffered from fibrovascular proliferation occurring in a region of the fundus that is rather uncommon in a diabetic eye. Secondly, that this complication occurred after cataract surgery, without previous vitrectomy is also unusual.

The development of anterior hyaloidal fibrovascular proliferation in diabetic eyes after cataract extraction, combined with the occurrence of a posterior capsular opacity in patients could be a challenge to the eye surgeon. Firstly, detailed viewing and examination of the fundus may be hindered by the posterior capsular opacity. Adequate retinal laser photocoagulation to treat this condition may be limited

${ }^{*}$ Correspondence: Dr. ON Okonkwo, Eye Foundation Retinal Institute, Ijebu Imushin, Ogun State, Nigeria • Email: o_okonkwo@yahoo.com 
by capsular opacity. Retinal cryotherapy to the peripheral retina may be an option. However, YAG laser capsulotomy may induce vitreous haemorrhage and accelerate the formation of rubeosis iridis and anterior segment neovascularization because angiogenic factors from the posterior segment can now cross the capsular barrier into the anterior segment. As more eye departments acquire a YAG laser machine and perform capsulotomies this complication may be encountered more often.

\section{AIM}

To report a rare case of anterior proliferative disease occurring in the form of anterior hyaloidal fibrovascular proliferation in a diabetic eye after cataract surgery. In this patient, the complication resulted in vitreous haemorrhage and hyphaema during YAG laser posterior capsulotomy. This patient had undergone cataract surgery with posterior chamber intraocular lens insertion 8 months earlier. This report also aims to sensitize eye surgeons who may be performing YAG laser procedures on diabetic eyes to be aware of the possibility of this complication occurring after cataract surgery and not only after vitrectomy procedure as is commonly observed and documented in published literature.

The importance of a satisfactory control of the blood sugar level and other systemic indices including the blood pressure, pre- and post-operatively is to be stressed in diabetic patients scheduled to undergo removal of cataract.

\section{CASE PResentation}

A 55-year-old male non insulin dependent diabetic (diagnosed 10 years prior to presentation) presented to the retina clinic on the $6^{\text {th }}$ of March 2006, with the complaint of a one-year history of cloudy vision in his left eye. He had noticed a gradual painless clouding of his left eye vision. Examination revealed right eye visual acuity of $6 / 9$ and left eye visual acuity of $6 / 36$ unaided. Left eye visual acuity was improved to $6 / 18$ with correction. Intraocular pressure (IOP) was $10 \mathrm{mmHg}$ in both eyes. No detailed view of the fundus could be seen in the left eye due to a visually significant cataract. An early cataract was noted in the right eye and funduscopy revealed right eye macular hard exudates and haemorrhage (fig. 1).

\section{Fasting Blood Sugar}

The patient had $337 \mathrm{mg} / \mathrm{dl}$ (markedly elevated)/BP: $160 / 90 \mathrm{mmhg}$ (systolic hypertension). The PCV was $32 \%$; urinalysis did show $4+$ glucose and $3+$ protein. No ketones were present in the urine. He was advised to institute strict control of blood sugar and see his physician for the control of systemic indices before surgery to remove cataract. He was seen again on the $18^{\text {th }}$ of April 2006 and confirmed that he had been certified fit for surgery by his attending physician.

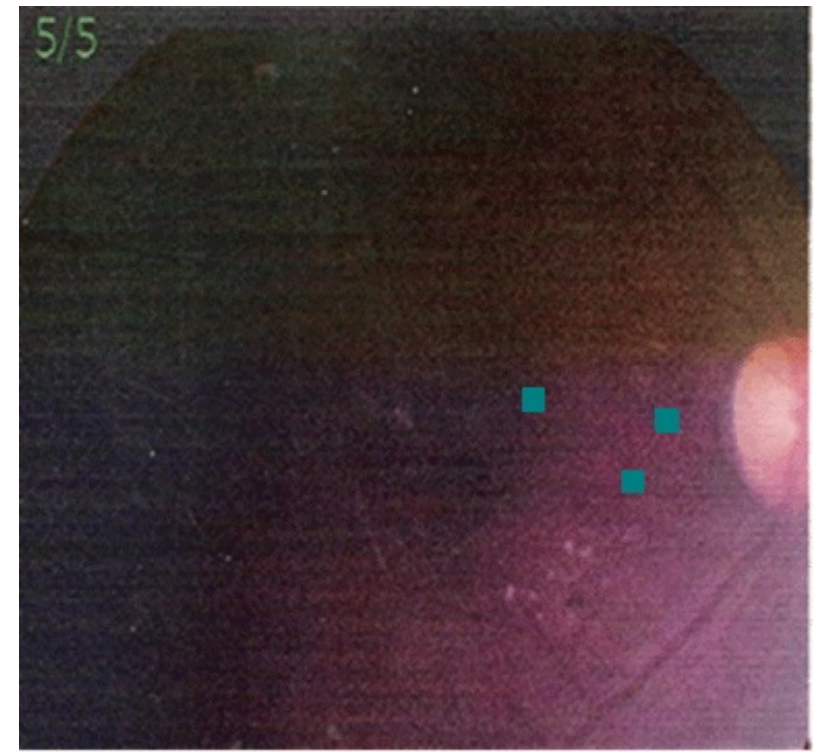

Figure 1. Showing right eye fundus with macular hard exudates and dot haemorrhage.

Subsequently, he underwent a left eye phacoemulsification of cataract and insertion of a posterior chamber intraocular lens. Intraoperatively, as the lens nucleus was hard and difficult to phacoemulsify completely, there was an intraoperative conversion to extracapsular cataract extraction, with wound extension and the remains of the nuclear fragment was delivered manually. Procedure was otherwise uneventful and the posterior lens capsule was intact. Post operative visual acuity at one month was 6/18 unaided and 6/9 aided. At this visit, clinically significant diabetic macular oedema was noted in the left eye (see fig. 2). He did undergo focal retina laser photocoagulation to this eye during the same visit (at the $1^{\text {st }}$ month post surgery).

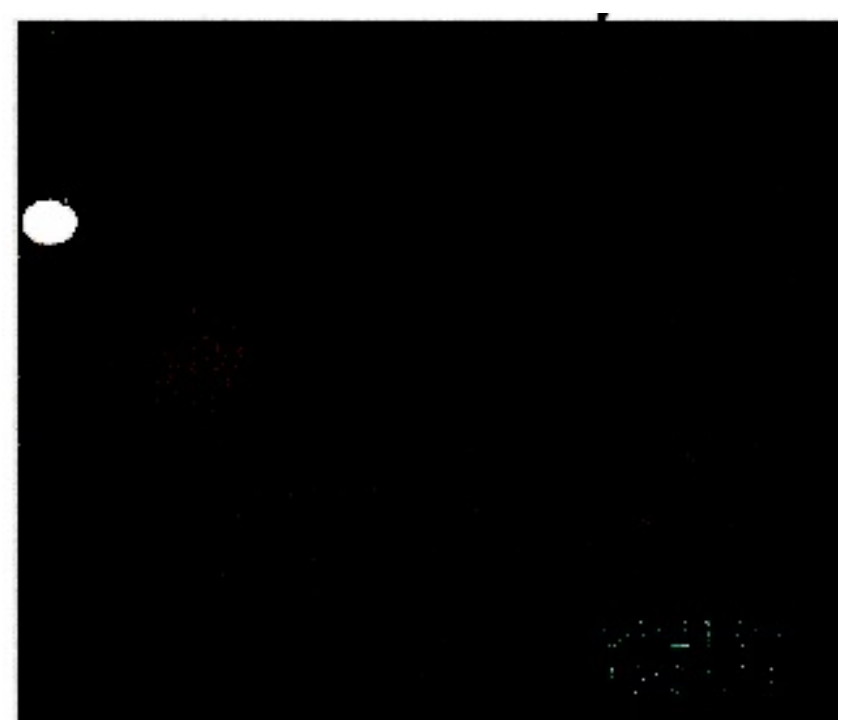

Figure 2. Showing left eye clinically significant macular oedema with a hard exudate. 
He defaulted on his clinic appointment and was seen 7 months later.

Then he complained of poor vision in the left eye. A significant posterior capsular opacification was noted behind the intraocular lens with vision of $6 / 18$ unaided and $6 / 18+$ with a pinhole in the left eye. His IOP was $18 \mathrm{mmHg}$ in the left eye on this visit. Upon direct questioning, he admitted to not having achieved a satisfactory glycaemic and alcohol intake control. There was no evidence of rubeosis on the iris surface.

YAG laser capsulotomy was performed, and during this procedure, haemorrhage was noted from a superior location behind the posterior capsule, into the anterior vitreous. This resulted in a vitreous haemorrhage and a diffuse hyphaema with reduction in visual acuity to PL.

Ultrasonography revealed a vitreous haemorrhage and anterior hyaloidal proliferation seen as echogenicity predominantly in the anterior vitreous, with a track of the haemorrhage extending from behind the anterior vitreous to the posterior vitreous.

On slit lamp examination, the gap created in the posterior capsule during the YAG laser procedure was covered by a blood clot.

Diffuse hyphaema cleared by the next day, but the vitreous haemorrhage persisted. Despite surgical efforts made at salvaging this eye, all vision was eventually lost.

\section{DISCUSSION}

Anterior hyaloidal fibrovascular proliferation has been reported as the most common postoperative complication following diabetic vitrectomy. In a group of 74 consecutive eyes that underwent vitrectomy for complications of diabetic vitrectomy. ${ }^{1}$

There have been only few reports of AHFVP occurring after cataract surgery. ${ }^{4}$

This case report provides evidence supporting the occurrence of this entity in not only post vitrectomized eyes, but following cataract surgery.

Clinical features of AHFVP include recurrent vitreous haemorrhages into the vitreous cavity or anterior vitreous or both vessels or fibrovascular tissue on the posterior lens capsule (this may have been the case in the patient presented, but vessels may not have been observed or were missed because of the capsular opacity), anterior extraretinal vascularization extending towards the lens on the anterior hyaloids (this hypothetically may also have occurred in this case), traction detachment of the peripheral retina or ciliary body and hypotony. ${ }^{1,3}$

AHFVP can be diagnosed with the use of ultrasound biomicroscopy. The echographic appearance has been well described. ${ }^{2,6,10}$
The usefulness of B Scan ultrasonography in identifying AHFVP in eyes at risk among diabetic patients who have a significant posterior capsule thickening following routine ECCE should be emphasized. A high index of suspicion is required and risk factors for this entity occurring after ECCE should be always borne in mind. ${ }^{4}$

The risk factors noted for the occurrence of anterior hyaloidal fibrovascular proliferation after cataract include proliferative diabetic retinopathy, iris neovascularization and anterior ischaemic retina. ${ }^{3}$ These three risk factors should serve as a caution to eye surgeons as they manage these patients. This patient had defaulted on his clinic visits and had not been attentive to proper glycaemic control during his postoperative period. It is likely he may have developed proliferative diabetic retinopathy which may not have been observed, as the examination of the fundus was limited by the posterior capsule opacity. This being the case, prompt retinal laser photocoagulation may have been helpful in the management of his clinical situation had he been seen before the development of the thickened posterior capsule. It then becomes important to point out the usefulness of more frequent and shorter postoperative clinic appointments for diabetic eyes with a suspicion of poor glycaemic control. The eyes should probably have had prompt and more aggressive retinal laser photocoagulation when proliferative disease was noted. Patients and surgeons should be aware of this, just as the importance of postoperative maintenance of strict glycaemic control by the physician should be stressed. The patient should maintain satisfactory control of his blood sugar and other systemic indices not only before the cataract surgery, but equally after the cataract surgery.

It is important to stress the following three points. First, identification of growing vessels or fibrovascular tissue on the posterior lens capsule after cataract surgery (as can be seen in AHFVP), in a patient who presents with a posterior capsule thickening may pose a considerable challenge. Secondly, the fibrovascular growth on the posterior lens capsule could also result in a worsening of the posterior capsular opacity, further obscuring the visualization of the growing vessels or fibrovascular tissue. Thirdly, an opaque posterior capsule would prevent adequate retinal examination and could prevent detection of proliferative diabetic retinopathy which is a recognized risk factor for the occurrence of AHFVP after cataract surgery. ${ }^{4}$

Caution should be used before scheduling this category of eyes with AHFVP for routine YAG laser capsulotomy. Such conditions will benefit more from aggressive retinal laser photocoagulation, or retinal cryotherapy (if the view of the fundus is obscured) or surgical management by a vitreoretinal unit. ${ }^{4}$ YAG laser capsulotomy in this group of patients with AHFVP can only result in vitreous haemorrhage and hyphaema, as seen in the case reported and should be avoided if possible. 
In the early stages of AHFVP, visual acuity may be unaffected. It is instructive to note that the patient still had a visual acuity of $6 / 18$ despite having this complication. Also, this complication had developed within 8 months following cataract surgery in a poorly controlled diabetic. This is a shorter duration than the 12-month average previously reported in 4 patients. ${ }^{3}$ This may have been as a result of the extremely poor control of the patients' blood sugar within the 8-month period, when he defaulted his clinic visit. A similar report of this complication occurring in 4 patients with proliferative diabetic retinopathy after ECCE and PCIOL noted that it affected the vision in only one of four patients. ${ }^{3}$ This case corroborates the findings that vision could still be good in a pseudophakia, despite this complication. With vision being unaffected early in the disease the patient may have no initial vision-related complaints during clinic visits. Yet it is at this early stage of the disease that treatment must be instituted if the eye is to be saved. Therefore, the predisposing factors for this disease must be borne in mind and the ophthalmic surgeon confronted with this category of patients should have a high index of suspicion for its occurrence.

Generally, this case points out the difficulties and challenges that could be encountered in a clinical situation of significant posterior capsule-thickening and occurrence of AHFVP following cataract surgery in diabetic eyes. A high index of suspicion should be borne in mind in diabetic patients who still have a poor control of blood sugar after cataract surgery. Appropriate attention should be given to the standard care of a diabetic, and good control of the blood sugar is required not only pre-operatively, but should be continued post-operatively. To manage this condition, if detected early in eyes with a clear media requires a timely additional extensive retinal laser photocoagulation applied to the posterior and anterior retina. ${ }^{3}$ Cryotherapy to the retina periphery can be useful if the media is hindered by a posterior capsular opacity.
YAG laser capsulotomy should be performed with caution in the setting of the AHFVP and a significant posterior capsule opacity as vitreous haemorrhage and hyphaema may complicate such cases.

\section{REFERENCES}

1. Lewis HH, Abrams GW, Williams GA. Anterior hyaloidal fibrovascular proliferation after diabetic vitrectomy. Am J. Ophthalmol. 1987; 104 (6): 607-13.

2. Bhende M, Agraharam SG , Gopal L, Sumasri K, Sukumar B, George J, et al. Ultrasound biomicroscopy of sclerostomy sites after pars plana vitrectomy for diabetic vitreous haemorrhage. Ophthalmology 2000; 107 (9):1729-36.

3. Eliott D, Lee MS, Abrams GW. Proliferative diabetic retinopathy: principles and techniques of surgical treatment. SJ Ryan, editor, Retina, $4^{\text {th }}$ ed,. Philadelphia, Mosby, 2006; 2440.

4. Ulbig MR, Hykin PG, Foss AJ, Schwartz SD, Hamilton PA. Anterior hyaloidal fibrovascular proliferation after cataract extraction in diabetic eyes. Am J Ophthalmol. 1993; 115: 321- 26.

5. Han DP, Pulido JS, Mieler WF, Johnson MW. Vitrectomy for proliferative diabetic retinopathy with severe equatorial fibrovascular proliferation. Am J Opthalmol.1995; 120.

6. Berinstein DM, Garretson BR, Williams GA. Ultrasound biomicroscopy in a case of anterior hyaloidal fibrovascular proliferation. Ophthalmic Surg Lasers 2000; 31(1): 69-70.

7. Michels RG. Proliferative diabetic retinopathy: pathophysiology of extraretinal complications and principles of vitreous surgery. Retina 1981; 1: 1-17.

8. Taylor E, Dobree JH. Proliferative diabetic retinopathy: site and size of initial lesions. Er J Ophthalmol 1970; 54: 11-8.

9. Davis MD. Vitreous contraction in proliferative diabetic retinopathy. Arch Ophthalmol 1965; 74:741 - 51.

10. Han DP, Lewandowski M, Mieler WF. Echographic diagnosis of anterior hyaloidal fibrovascular proliferation. Arch Ophthalmol 1991; 109: 842-6. 